Instructions for authors, subscriptions and further details:

\title{
http://mcs.hipatiapress.com
}

\section{The Social Life of Gender}

Vladia Ionescu ${ }^{1}$

1) Universidad de Barcelona, Spain

Date of publication: October $21^{\text {th }}, 2018$

Edition period: October 2018-February 2019

To cite this article: Ionescu, V. (2018). The Social Life of Gender. [Review of the book]. Masculinities and Social Change 7(3), 313-314. doi:

10.17583/MCS.2018.3806

To link this article: http://dx.doi.org/10.4471/MCS.2018.3806

\section{PLEASE SCROLL DOWN FOR ARTICLE}

The terms and conditions of use are related to the Open Journal System and to Creative Commons Attribution License (CC-BY). 

MCS - Masculinities and Social Change Vol. 7 No. 3 October 2018

pp. 313-314

\section{Reviews (I)}

Ray, R., Carlson, J., \& Andrews, A. (Eds.). (2017). The Social Life of Gender. New York: Sage Publication

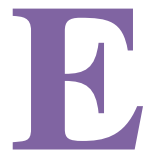

stamos ante un libro que presenta un análisis profundo sobre la masculinidad en dos países con tradición en este ámbito de estudio: Estados Unidos y Reino Unido. Además, la obra enfrenta dos paradigmas en los que se han movido las ciencias sociales y en especial, los men's studies en la era contemporánea: "la corporalidad" y "los sistemas". De esta forma se plantea la construcción social del género y su impacto en la estructura social, pero también se tiene en cuenta el efecto en las identidades y cuerpos de los sujetos.

En el libro hay diferentes capítulos donde se aborda el estudio de la masculinidad en Estados Unidos, haciendo hincapié, por ejemplo, en la figura del cowboy que tanto peso tiene en la socialización de género en este país. En otro capítulo también se aborda el influjo que tiene el ejército y el sector militar en la construcción de la identidad masculina. En este sentido, existe un capítulo donde se analiza el efecto que tuvieron los atentados del 11 de Septiembre del 2001, aprovechando algunas representaciones como la del libro Saturday de Ian McEwans y la película World Trade Center del director Oliver Stone. De hecho, como ya han hecho otras obras alrededor de los estudios de masculinidad, el libro incluye otros capítulos donde también se exploran las representaciones de artículos como el best seller The Dogs of War donde se presenta el heroismo a las "masculinidades mercenarias".

En el libro se estudian otras representaciones de la masculinidad que aparecen en diferentes libros, series y películas. En este sentido, resulta interesante el análisis que se efectúa sobre la serie de HBO Deadwood, la obra de William Gaddis o las representaciones cinematográficas sobre masculinidad, en películas recientes, que abordan la realidad de Wall Street.

2018 Hipatia Press

ISSN: 2014-3605

DOI: $10.17583 / \mathrm{MCS} .2018 .3806$ 


\section{Ionescu-The Social Life of Gender [Book Review]}

Resulta interesante también la incorporación de una pieza de Michael Kimmel, autor de referencia en el ámbito de las masculinidades, que habla sobre la supremacia blanca - y del hombre blanco en particular- en los Estados Unidos.

En definitiva, el libro nos aproxima a investigaciones recientes en el campo de las masculinidades que nos ilustran diferentes ejemplos de representaciones contemporáneas alrededor de la masculinidad. También nos enseña la existencia de estereotipos y disciminaciones que no podemos olvidar y que continúan perpetuando el esquema patriarcal de hombre blanco y heterosexual como representación hegemónica. Así, los autores y autoras de esta obra plantean unas líneas de trabajo futuras más que interesantes para seguir avanzando en la literatura científica de los men's studies.

Vladia Ionescu, Universidad de Barcelona. vladia.ionescu@gmail.com 